\title{
Factors associated with children's health facility visits for primaquine treatment in rural Papua New Guinea
}

Akiko Yoshii ${ }^{1,2}$, Makoto Sekihara ${ }^{2,3}$, Takuro Furusawa ${ }^{4}$, Francis Hombhanje ${ }^{5}$ and Takahiro Tsukahara ${ }^{2,6^{*}}$ (1)

\begin{abstract}
Background: To control and eventually eliminate vivax malaria, radical treatment with primaquine $(P Q)$ is essential after completion of blood-stage treatment. Although in many malaria-endemic countries, village health volunteers (VHVs) are engaged in diagnostic treatment of malaria in remote communities, they principally provide blood-stage treatment. In such a situation, access to PQ following blood-stage treatment can be a barrier to complete treatment. However, studies on access to PQ treatment have been scarce and limited in health facility-based settings. This study aimed to identify factors associated with access to PQ treatment in rural Papua New Guinea (PNG) from the community case management perspective.
\end{abstract}

Methods: A community-based, cross-sectional survey was conducted to collect sociodemographic information on children under 15 years of age, their households, and their caretakers in East Sepik Province, PNG. Data collection lasted from February to March, 2015. Information on the diagnoses of potential non-falciparum malaria and prescription of PQ in preceding year (January to December 2014) were obtained from child health-record books. Then, multilevel logistic regression model was used to determine the factors associated with formal health facility visits for $\mathrm{PQ}$ treatment among children with potential non-falciparum malaria.

Results: Of 420 episodes diagnosed as potential non-falciparum malaria, 46 (11\%) were immediately given PQ. The rest were instructed to visit formal health facilities (HFs) for $P Q$, and the patients obtained $P Q$ during the second visit to HFs was $44 \%$. Consequently, the overall proportion of PQ prescription was 50\%. Logistic regression analysis suggested that among the patients who were instructed to visit HFs for PQ treatment, the initial visit to VHV and higher transportation costs to HF were inversely associated with PQ prescription during the second visit to an HF.

Conclusions: Few children received PQ treatment during the second visit to HFs following diagnosis of potential non-falciparum malaria. These findings suggest a need to establish a policy to reduce structural and economic barriers and improve rural inhabitant access to PQ treatment.

Keywords: Antimalarials, Therapeutic uses, Child, Vivax malaria, Continuity of patient care, Health service accessibility, Patient compliance, Patient acceptance of health care, Appointments and schedules

\footnotetext{
*Correspondence: tsukahara.takahiro@twmu.ac.jp

${ }^{2}$ Department of International Affairs and Tropical Medicine,

Tokyo Women's Medical University, 8-1 Kawada-cho, Shinjuku-ku,

Tokyo 162-8666, Japan

Full list of author information is available at the end of the article
} 


\section{Background}

With the global effort to control malaria, the relative proportion of Plasmodium vivax tends to increase in regions where $P$. vivax and Plasmodium falciparum are co-endemic [1]. In countries outside Africa, vivax malaria accounts for $36 \%$ of total malaria cases [2]. Unlike $P$. falciparum, $P$. vivax can remain dormant in the liver as hypnozoites, reemerging to cause recurrent symptoms and new transmissions, which comprises a major obstacle to control this disease. Primaquine (PQ) is the most standard and approved efficacious drug used against hypnozoites.

In Asia-Pacific countries, $P$. vivax is dominant and the region accounts for over $80 \%$ of $P$. vivax cases other than Africa [3]. In 2009, the Asia-Pacific Elimination Network (APMEN) was established especially focusing on controlling $P$. vivax with 18 affiliate countries [4]. Although, in all member states, PQ has been introduced as a component of the standard treatment of vivax malaria [2], information on the effectiveness of PQ treatment in routine clinical settings is sparse. Previous studies have focused mainly on adherence to 14 days' administration of PQ with varied adherence rate from 24 to $98 \%$ [5-8]. In addition, these studies showed that males, children, side effects, oral instruction for treatment, and improvement or worsening of symptoms tended to be related to nonadherence.

In many malaria-endemic countries, village health volunteers (VHVs) are often the first point of contact with medical services. Among APMEN countries, 11 countries have deployed VHVs for malaria diagnosis and treatment in remote communities [9-19]. However, the VHVs have generally engaged in blood-stage treatment of vivax malaria, and have not been allowed to prescribe PQ because of safety concerns about severe acute haemolytic anaemia in glucose-6-phosphate dehydrogenase (G6PD)deficient patients. Although the possibility of PQ delivery by VHVs was discussed in the international meeting for vivax elimination in 2016, the current guideline of the World Health Organization (WHO) does not endorse this option [4].

In Papua New Guinea (PNG) one of the APMEN countries, the different Plasmodium species co-exist, and $P$. vivax is a major contributor to high morbidity rates for malaria, especially in young children $[20,21]$. The incidence of $P$. vivax malaria is seasonal and reaches a peak in the early rainy season, from October to November [21]. A new national malaria-treatment protocol was officially announced in 2009, and parasite-based diagnoses using microscopy or rapid diagnostic testing became required instead of symptom-based diagnoses. In our previous study in PNG, of the febrile children who sought treatment outside the home, $60 \%$ had first consulted
VHVs [15]. However, PQ has been provided only in formal health facilities where health providers can clinically assess a patient for potential PQ-induced haemolysis, and children diagnosed with vivax malaria are instructed to visit HFs to obtain PQ after blood-stage treatment with artemether-lumefantrine (AL) in a community level.

In such a situation, access to PQ treatment during the second visit to an HF following blood-stage treatment can be a barrier to complete the treatment. However, previous studies on access to PQ treatment have been scarce, and limited in health facility-based settings [2224]. Therefore, access to $P Q$ treatment was investigated, including referrals from VHVs to HFs in an actual lowresource setting, as well as associated factors from the community case management perspective, to propose policy recommendations for controlling vivax malaria in the Asia and Pacific region.

\section{Methods \\ Study site}

The study was conducted in the Boiken Dagua Rural Local Level Government area of Wewak district, located on the northern coast of East Sepik Province, PNG. In East Sepik Province, $P$. vivax is the second-most prevalent parasite after $P$. falciparum; the overall mean prevalence of P. vivax was $35.7 \%$ [20]. In PNG, non-governmental organizations have promoted the deployment of VHVs since the early 2000s [25-27]. There were 23 villages in the study area, and one VHV was deployed in each village. Details of VHV framework have been previously described [28]. The term "health facility" (HF) was used to define a formal facility operated by health professionals approved to prescribe PQ. At the time of this study, five HFs (one health centre and four aid posts) were operated in the study area.

High-grade $P$. vivax chloroquine (CQ) resistance was reported in PNG in the 1990s [29, 30], therefore, combinations of $\mathrm{CQ}$ or amodiaquine with sulfadoxinepyrimethamine (SP) for $P$. vivax were introduced in 2002. Because of the subsequent rapid increase in SP resistance, a drug efficacy trial comparing AL, dihydroartemisinin-piperaquine (DHA-PPQ), and $\mathrm{CQ}+\mathrm{SP}$ was conducted between 2005 and 2007 [31]. This showed that the $\mathrm{AL}$ regimen was the most efficacious against $P$. vivax, followed by the DHA-PPQ, and $\mathrm{CQ}+\mathrm{SP}$ regimens. For these reasons, AL became a first-line treatment for vivax malaria in the new national malaria-treatment protocol in 2009 [32].

Since then, $\mathrm{PQ}$, which the former guideline recommended only for vivax malaria patients in low malaria-endemic regions [33], has become a standard treatment for all patients diagnosed with uncomplicated vivax and/or ovale malaria after completing 3 days of 
treatment with AL. If a patient's G6PD deficiency status is unknown, health providers should advise the patient to stop PQ and return to the health facility if they pass darkcoloured urine, or they should clinically assess the patient for a minimum of $1 \mathrm{~h}$ following the administration of PQ [34]. Following WHO recommendations [35], a dose of $0.25 \mathrm{mg} / \mathrm{kg}$ daily for 14 days was adopted for radical cure of $P$. vivax in the PNG guideline. In the study area, a rapid diagnostic test (RDT; CareStart $^{\mathrm{TM}}$ Malaria HRP2/pLDH (pf/PAN) Combo, Access Bio Inc., USA) was widely used instead of microscopic diagnosis, and PQ treatment was recommended for patients diagnosed with "potential non-falciparum malaria", i.e., "a non-P. falciparum," "P. falciparum, or P. falciparum with another species."

\section{Study population and data collection}

A community-based, cross-sectional survey using a structured questionnaire was conducted during February and March 2015. The study population included children aged 0 to 14 years living in coastal lowlands of the catchment area of Dagua Health Centre. The area consisted of 23 villages with 1056 households and 2767 children. A structured questionnaire was used for collecting information, and it was written in English (one of the official languages of PNG). Coauthors (TT, MS and TF) were able to communicate in the local language (Tok Pisin: lingua franca and another official language of PNG) and they instructed field assistants on the intended meaning of each question and how to administer the questionnaire in Tok Pisin. Trained field assistants interviewed caregivers of the target children to collect sociodemographic information. Medical records of malaria diagnosis results and subsequent treatment regimens for study participants during the preceding year were also collected (January to December 2014). Individual outpatient records were not kept in the study area's HFs. Instead, VHVs, as well as medical personnel of HFs are ordered to fill out medical information in a child health-record book, which each patient retained at home and was expected to bring to each VHV/HF visit. For that reason, trained field assistants visited each household and collected child healthrecord books; the relevant pages from these books were photocopied and then returned to the caregivers. Then, the necessary information for the study was encoded and was single-entered into a database table.

\section{Data analysis}

Following local guidelines, episodes with potential nonfalciparum malaria were included for the logistic regression analysis. The dependent variable was "access to PQ", defined as the PQ prescription during the second visit to HFs following diagnosis of potential non-falciparum malaria. The child's gender, child's age, caregiver's age, caregiver's education level, caregiver's marital status, total number of residents in the household, durable assets in the household, place of initial visit (an HF or a VHV), and transportation costs were identified as independent variables.

In this study, the caregiver's education level was specified as the total years of schooling. Household size was defined as the total number of residents in the household. On the basis of 13 dummy asset variables (pig, chicken, clock, mobile phone, radio, generator, chain saw, solar panel, car, bicycle, computer, television, and refrigerator), an asset index, as a predictor of household economic status, was calculated using principal component analysis (PCA) [36, 37]. The asset index was scored on the basis of the first principal component. Thereafter, these scores were divided equally into three categories: upper, middle and lower.

Transportation cost was defined as the cost of return fare of transport vehicle from the nearest roadside to HFs. There was no charge to receive $P Q$, including related consultation and treatment costs, at HFs, so the direct cost to patients and their caregivers equaled only transportation cost.

All data were entered into a Microsoft Access 2013 database and were then analysed using Stata SE14 (Stata Corp., College Station, TX, USA). After the characteristics of the children, caregivers, and households surveyed were entered, a three-level multilevel random intercept logistic regression model was used to identify the association between PQ prescription during the second visit to an HF and those characteristics. In the analysis, potential non-falciparum malaria episodes (level 1) were nested in an individual (level 2) and individuals were nested in a village (level 3). Wilcoxon rank-sum tests were used to compare medians of continuous variables, and Chisquare tests were applied to compare proportions of categorical variables between the groups. The level of statistical significance was set at $p<0.05$.

\section{Results \\ Potential non-falciparum malaria episodes for multilevel logistic regression analysis}

This study population included 2767 children under age 15 registered in the household questionnaire survey. Of these, $30 \%(835 / 2767)$ were excluded because of uncollected child health-record books: children had no book (16\% [437/2767]); children had moved out of the community at the time of record-book collection (11\% [312/2767]); caregivers declined to provide a child health-record book (3\% [86/2767]). Descriptive statistics of the characteristics of children in the excluded group $(n=835)$ were compared with those in the remaining group $(n=1932)$. There were significant differences in 
the age of the patients, the age of the caregivers, the marital status of caregivers, household size, and wealth status between the two groups (Additional file 1: Table S1).

Among the remaining 1932 children, 684 were examined for malaria infection using RDT at least once during the study period. A total of 1059 RDTs were performed. Of these, 639 (60\%) were excluded (77 had a P. falciparum single infection, 437 had negative RDT results, 125 had improper or unrecorded RDT or PQ prescription results). Among the remaining 420 episodes diagnosed as potential non-falciparum malaria, $50 \%(210 / 420)$ were prescribed PQ including 46 episodes $(11 \%)$ which were directly prescribed PQ at their initial medical care. Finally, 374 potential non-falciparum malaria episodes were selected for the logistic regression analysis (Fig. 1).

\section{$\mathrm{PQ}$ prescription during the second visit to $\mathrm{HF}$}

Of 374 potential non-falciparum malaria episodes in which children were directed to revisit HFs for PQ treatment, 164/374 (44\%) were prescribed PQ during the second visit to HFs (Fig. 1). The median between the first visit (when blood-stage treatment with AL commenced) and the second visit to HFs (when patients obtained PQ treatment) was 4 days (interquartile range [IQR] 3, 6) in 164 episodes. Among 210 episodes with no PQ prescription, four visited HFs within 14 days after diagnosis of potential non-falciparum malaria. Some children had multiple potential non-falciparum malaria episodes during the study period. On average, 1.2 potential non-falciparum malaria episodes per child (374 episodes in 309 children) were seen, with a maximum of three episodes in one child.

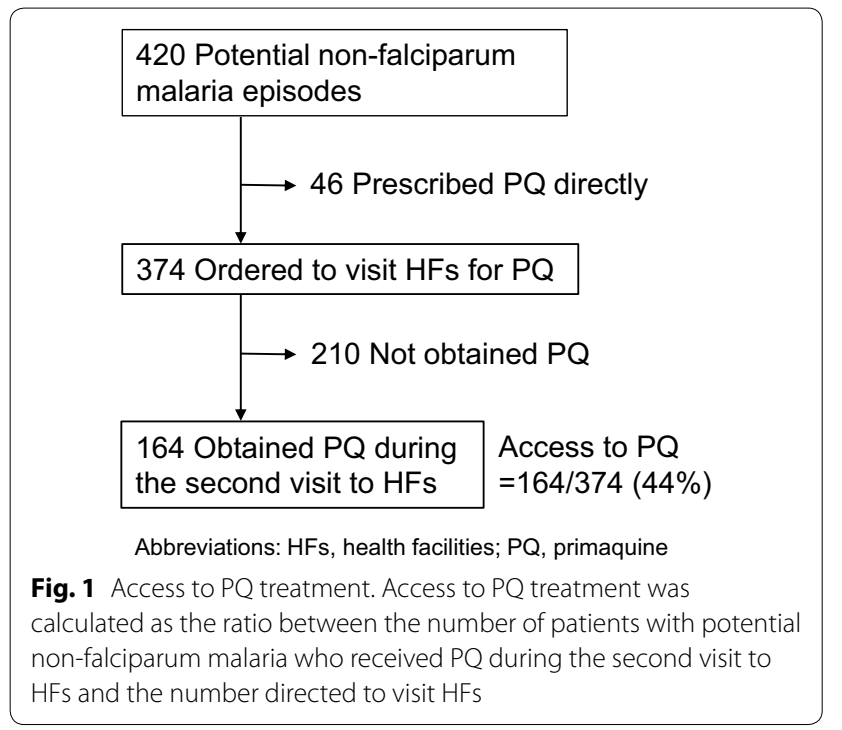

\section{Characteristics of children, their caregivers, and their households}

Descriptive statistics of the characteristics of children included in the analysis are shown in Table 1. Of the 309 children who received at least one diagnosis of potential non-falciparum malaria, $54 \%$ were boys, and the median age was 7 years (IQR 4, 10). Most of caregivers were married $(92 \%)$, and the median caregiver age was 34 years (IQR 28, 42). The median number of years of education was 6 (IQR 6, 8). The median household size was 6 (IQR 5 , 7), with a maximum of 17 people in one household. The median transportation cost to HFs was 0 kina ([IQR 0 , 4], Kina $1=$ USD 0.37 in 2015).

Determinants of $P Q$ prescription during second visit to $\mathrm{HF}$ Using a three-level multilevel random intercept logistic regression model, place of initial visit and transportation cost to HFs were factors significantly associated with obtaining a PQ among children with potential non-falciparum malaria. Patients who visited VHVs at initial visit

Table 1 Sociodemographic characteristics of children with potential non-falciparum malaria who were directed to visit an $\mathrm{HF}$ for $P Q$

\begin{tabular}{ll}
\hline Variable & Value \\
\hline Gender of patients—n (\%) & \\
Male & $167(54.0)$ \\
Female & $142(46.0)$ \\
Age of patients, years—-median [IQR] & $7[4-10]$ \\
Age of caregivers, years—-median [IQR] & $34[28-42]$ \\
Educational level of caregivers, years—-median [IQR] & $6[6-8]$ \\
Marital status of caregivers—n (\%) & \\
Married & $286(92.6)$ \\
Unmarried & $6(1.9)$ \\
Divorced or separated & $10(3.2)$ \\
Widowed & $7(2.3)$ \\
Household size-median [IQR] & $6[5-7]$ \\
Missing— $\mathrm{n}$ & 1 \\
Wealth status-n (\%) & \\
Lower & $107(36.0)$ \\
Middle & $90(30.3)$ \\
Upper & $100(33.7)$ \\
Missing & 12 \\
Transportation cost to HFs, Kina —-median [IQR] & $0[0-4]$ \\
Missing—n & 4 \\
Place of initial visit—n (\%) & \\
VHV & $168(54.4)$ \\
HF & $141(45.6)$ \\
\hline
\end{tabular}

Shapiro-Wilk's normality test found all continuous variables to be non-normally distributed

$V H V$ village health volunteer, $H F$ formal health facility, IQR interquartile range

a PNG Kina $1=$ USD 0.37 in 2015 
were less likely to be prescribed PQ during the second visits to HFs (OR $=1.92,95 \%$ CI $1.00-3.69, p=0.049)$, and higher transportation cost to HFs was associated with lower odds of PQ prescription ( $\mathrm{OR}=0.85,95 \% \mathrm{CI}$ $0.76-0.94, p=0.002$ ) (Table 2).

\section{Discussion}

The study's main findings were as follows. First, the overall proportion of patients with potential non-falciparum malaria who received PQ was $50 \%$. Second, the proportion of patients who received PQ during the second visits to HFs was $44 \%$. Third, a multilevel logistic analysis suggested that the initial visit to a VHV and higher transportation costs were inversely associated with the PQ prescription during the second visit to an HF.

The $50 \%$ proportion of patients who received PQ was lower than that found by previous studies in the Peruvian Amazon (78\%) [22] and in Indonesia (65\%) [23]. However, these studies have investigated proportions of patients who received PQ treatment from medical records or patients' self-reports in medical facilities where patients visited. Thus, cases of referral patients are not included, and this may have resulted in a higher proportion of patients who reached PQ treatment.

The present study found children diagnosed with potential non-falciparum malaria in HFs were more likely to obtain PQ during their second HF visit than those diagnosed by VHVs (Table 2). These results may have

\begin{tabular}{lll}
$\begin{array}{l}\text { Table } 2 \text { Three-level } \\
\text { associated with PQ prescription } \\
\text { to HF }\end{array}$ & $\begin{array}{c}\text { regression } \\
\text { during the second visit }\end{array}$ \\
\hline Variable & OR (95\% Cl) & p-value \\
\hline Gender of patients (female=0/male=1) & $1.23[0.68,2.23]$ & 0.49 \\
Age of patients (year) & $1.05[0.96,1.15]$ & 0.31 \\
Age of caregivers (year) & $1.03[0.99,1.06]$ & 0.16 \\
Educational level of caregivers (year) & $1.02[0.92,1.14]$ & 0.66 \\
Marital status of caregivers (married $=1)$ & & \\
$\quad$ Unmarried & $1.08[0.17,6.88]$ & 0.94 \\
$\quad$ Divorced/separated & $1.78[0.37,8.52]$ & 0.47 \\
$\quad$ Widowed & $0.25[0.03,2.19]$ & 0.21 \\
Household size & $0.92[0.81,1.04]$ & 0.19 \\
Wealth status (Lower $=1)$ & & \\
$\quad$ Middle & $0.82[0.40,1.68]$ & 0.59 \\
$\quad$ Upper & $0.73[0.35,1.52]$ & 0.41 \\
Transportation cost to HF (PNG Kina) & $0.85[0.77,0.94]$ & 0.002 \\
Place of initial visit (VHV=0/HF=1) & $1.94[1.01,3.71]$ & 0.047
\end{tabular}

Transportation cost to the $\mathrm{HF}(p=0.002)$ and place of initial visit $(p=0.047)$ were significantly affected $P Q$ prescription during the second visit to an HF. The significance level was $p<0.05$

$V H V$ village health volunteer, $H F$ health facility, $O R$ odds ratio, $C I$ confidence interval been due to VHV's inadequacy of specific instructions to patients. In PQ treatment-adherence studies in the Brazilian Amazon, patients who received adequate prescription instruction by health-care providers were more likely to adhere to treatment [38]. In the study area, VHVs did not receive refresher training after they completed initial short-term training for malaria case management. In addition, VHVs were not authorized to prescribe PQ, whereas HF health-care providers prescribe PQ as part of their daily work, so VHVs may not properly explain to the patients about the importance of the visit an HF for PQ. Moreover, disease severity may affect HF visits for PQ. Several studies showed that treatment seeking in the formal health sector was associated with disease severity $[39,40]$, and from that perspective, more severe patients may consult with HFs than VHVs and are likely to continue PQ treatment at HFs.

In the present study, higher transportation costs were negatively associated with $\mathrm{PQ}$ prescription during the second visit to an HF (Table 2). Transportation costs may substantially impact the study population and pressure people to sacrifice other living expenses. Although no studies have evaluated the effect of transportation costs on access to PQ treatment, several have shown association between transportation cost and appropriate treatment-seeking behaviour in an acute phase [41, 42]. In addition, this aligns with results from previous studies focusing on retention rates and transportation costs among patients with human immunodeficiency virus (HIV) in rural Haiti and Uganda [43, 44].

To improve access to HFs for PQ, structural and economic barriers may be critical elements to consider. Thus, four possible policy options are proposed: (1) decentralizing medical care by enabling VHVs to prescribe PQ, (2) free public transportation to HFs, (3) PQ provision through routine outreach activities, and (4) concurrent prescription of AL and PQ.

First, decentralization of PQ treatment could be a promising solution. In rural PNG, VHVs have been identified as a key basic health-care provider and have diagnosed and treated acute malaria patients. VHVs are the most accessible of health-care personnel for residents; therefore, empowering them to prescribe PQ could be an efficient and feasible way to improve access. For VHVs, a decentralized treatment of PQ would give greater responsibility in treating patients with vivax malaria. Among residents, this practice could increase interest about and understanding of PQ. Additionally, VHVs that are closer to residents have potential to more effectively improve a therapeutic course via directly observed therapy, such as medication adherence and monitoring risk of haemolysis among people with G6PD deficiency. However, in terms of G6PD deficiency, as haemolytic risk is considered to 
be high in PNG $[45,46]$, sufficient preliminary assessment of tolerability and safety of PQ administration must first be conducted. In cases where nonmedical workers intervene in PQ treatment, screening using point-of-care G6PD testing is recommended for the risk management of haemolysis in low-resource settings [47].

Second, free transportation could be a helpful incentive to revisit HFs. Currently, in PNG, examinations and PQ treatment are free at HFs, so the only direct cost for patients and their families is transportation. In a study conducted in Tanzania, funding assistance to cover the transportation cost of returning to the hospital was associated with improved adherence to follow-up protocols among children diagnosed with tuberculosis [48]. Similarly in Malawi, transportation cost to a hospital was associated with improved long-term follow-up in antiretroviral treatment among adult HIV patients co-infected with tuberculosis [49]. However, cost-effectiveness assessment, including management cost, would be necessary for the perspective of sustainability in PNG.

Third, the availability of routine outreach activities could improve PQ treatment for rural residents. In the study area, childcare-outreach services visit each village every 2 months to administer vaccinations and health examinations for children, providing PQ treatment as part of the outreach services may therefore be partly effective for reducing vivax transmission. PNG has a short-term relapsing strain in which multiple relapses are very common (similar to the Chesson strain; with which $80 \%$ of relapses occur within 1 month after the treated primary infection) [50]. Even if PQ treatment is given more than 2 months after the disease onset by the outreach services, it is still possible to reduce the number of relapses and infection transmission. Furthermore, during routine visits to each village, health-care professionals can monitor the signs and symptoms of possible adverse reactions for patients already given PQ treatment, and can also mentor the VHVs and share with them relevant health-care information on PQ-induced adverse reactions. These policies may lead to better implementation of treatment for needy children infected with vivax malaria.

Last, concurrent prescription of AL and PQ, which has been adopted in Vanuatu could increase the proportion of patients obtaining $P Q$ treatment: $P Q$ is prescribed with AL once and started on the same day with AL. Vanuatu's national guidelines recommended the routine testing of G6PD deficiency before PQ administration, and require clinical monitoring at higher level health facilities for patients with an unknown G6PD status at the time they received PQ treatment [51]. However, concurrent prescription needs some consideration. Eventual adherence, including 14 days oral administration, is unclear unless differences are compared between patients prescribed $\mathrm{PQ}$ at the initial visit and at the second visit. Moreover, little is known about the safety and efficacy of concurrent use of AL and PQ. Some partner blood schizonticides, including AL may affect PQ metabolism via inhibition of cytochrome P450-2D6 activity [1, 52]. For these reasons, health facilities should establish emergency responses to G6PD deficiency, such as blood transfusion for an acute haemolytic event. Furthermore, patients should be given instructions about the signs and symptoms of possible adverse reactions (such as dark-coloured urine, gastrointestinal symptoms and fatigue), emphasizing the necessity of a return visit if the patient experiences any of those adverse reactions.

Apart from the possible changes in policy as mentioned above, current PQ administration in the study setting may be problematic; it was found that PQ was prescribed for patients with an RDT result of "falciparum malaria or falciparum malaria with infections of other malaria species", although the national guidelines recommend that PQ should be prescribed only when confirmed as vivax and/or ovale malaria [34]. Therefore, the observed PQ treatment may lead to overuse of $\mathrm{PQ}$ and any subsequent unnecessary adverse reactions. It is rather preferable to establish a referral system to ensure a definitive diagnosis using a microscope or $P$. vivax-specific bivalent RDT toward the elimination stage of vivax malaria. In the present study setting, it is feasible to make a blood film diagnosis for malaria at the HF and refer to the provincial hospital for microscopic examination. In this situation, it is more important to strengthen a referral system between VHVs and HFs for elimination stage of vivax malaria at a community level.

The present study has some limitations. First, PQ prescription during the second visits to HFs among children with potential non-falciparum malaria was assessed through inspection of child health-record books. Possibly, health-care providers did not always enter patients' information in record books, and therefore, the proportion of children completing the requisite HF visits for PQ could be biased. However, this study is perceived to be more credible than a previous study evaluating the proportion of PQ prescriptions through patients' self-reports [23]. Second, access to PQ treatment was defined as obtaining PQ during a second HF visit following diagnosis of potential non-falciparum malaria. The word "access" originally has three dimensions, including availability, affordability, and acceptability [53]. However, the present study mainly focused on the former two aspects, excluding acceptability-such as perception of malaria disease and its severity, belief in the effectiveness of medicine, knowledge and perception of the risk of its adverse reactions, characteristics of health providers, 
and physician-patient relationships-that could affect the second visit to HF and subsequent PQ prescription. Third, indirect costs (the total costs of travel, waiting, and consultation time) could not be included in the analysis because it was not possible to obtain sufficient information to estimate these costs. The effect of costs on the PQ access may be overestimated. Fourth, the descriptive statistics of several explanatory variables showed significant differences between the excluded and the remaining groups, although there was no significant difference in the median of the significant determinant of $P Q$ access (i.e., the transportation cost to the HFs). This could have resulted in a selection bias on the results of the logistic regression analysis. Fifth, because not all communities in rural area of PNG have working VHV programmes, generalizing these results to the rest of the region might be difficult. However, this study might provide useful information for policy planning in regions that face similar problems.

Despite these limitations, the present study showed that there is inadequate access to PQ treatment in rural areas of PNG. Given the continuing global effort toward eliminating vivax malaria, further studies exploring PQ treatment from the stand point of community case management is important where VHVs are often the first contact point, which could contribute to establishment of better strategies for vivax malaria elimination in the Asia and Pacific region.

\section{Conclusions}

Few children obtained PQ treatment during their second visits to HFs following diagnosis of potential non-falciparum malaria in rural PNG. These findings suggest that inadequate referrals from VHVs to HFs, and economic barriers of traveling to HFs, were critical factors in the access to PQ treatment. Vivax malaria-control policy making should focus on ways to increase access to PQ, such as the decentralization of treatment, free transportation, enhancement of outreach activity, and concurrent prescription of $\mathrm{AL}$ and $\mathrm{PQ}$.

\section{Additional file}

Additional file 1: Table S1. Comparison of descriptive statistics between the remaining and the excluded groups.

\section{Abbreviations}

AL: artemether-lumefantrine; HF: formal health facility; HIV: human immunodeficiency virus; MHC: mobile health clinic; PCA: principal component analysis; PNG: Papua New Guinea; PQ: primaquine; RDT: rapid diagnostic test; VHV: village health volunteer; Cl: confidence interval; WHO: World Health Organization.

\section{Authors' contributions}

AY contributed to conceiving the study concept, designing the study, collecting and analysing the data, drafting the manuscript, and completing the final version. TT contributed to designing the study and to collecting and analysing the data. MS, TF, and FWH contributed to collecting data. All authors interpreted the results, revised the draft collaboratively. All authors read and approved the final manuscript.

\section{Author details}

${ }^{1}$ TWMU Career Development Center for Medical Professionals, Tokyo Women's Medical University, 8-1 Kawada-cho, Shinjuku-ku, Tokyo 162-8666, Japan. ${ }^{2}$ Department of International Affairs and Tropical Medicine, Tokyo Women's Medical University, 8-1 Kawada-cho, Shinjuku-ku, Tokyo 162-8666, Japan. ${ }^{3}$ Department of Tropical Medicine and Parasitology, Juntendo University School of Medicine, 6th Floor, Bldg. No.9, 2-1-1, Hongo, Bunkyo-ku, Tokyo 113-8421, Japan. ${ }^{4}$ Department of Ecology and Environment, Graduate School of Asian and African Area Studies, Kyoto University, Room\#AA431, Research Bldg. No.2, Yoshida-Honmachi, Sakyo-ku, Kyoto 606-8501, Japan. ${ }^{5}$ Centre for Health Research and Diagnostics, Divine Word University-Rabaul Campus, Kokopo, Papua New Guinea. ${ }^{6}$ School of Economics, Hosei University Graduate School, 2-15-2 Ichigaya Tamachi, Shinjuku-ku, Tokyo 162-0843, Japan.

\section{Acknowledgements}

This study was a collective effort involving many partners. The authors thank all study participants including the VHVs, caregivers, children, the Catholic Church Health Services of Wewak Diocese, the Division of Health of East Sepik Province, and Wewak General Hospital for conducting the field survey. The authors are grateful to Dr. Jun Baba and Mr. Hikaru Hamaguchi for the assistance with data collection, to Mr. Daichi Takahashi for data collection and data input, and Dr. Katsunori Shimada for his advice on the questionnaire design. The authors would like to thank Enago (http://www.enago.jp) for the English language review.

\section{Competing interests \\ The authors declare that they have no competing interests.}

\section{Availability of data and materials}

The datasets used and/or analysed in the current study are available from the corresponding author on reasonable request.

\section{Consent for publication}

No individual data or comments are contained in this paper.

\section{Ethics approval and consent to participate}

This study was approved by the Medical Research Advisory Committee of the Papua New Guinea National Department of Health (No. 14.22) and the Tokyo Women's Medical University Ethical Committee (No. 1744). It was conducted in accordance with the Declaration of Helsinki and the recommendations of those committees, with written informed consent obtained from all participants. Oral informed consent from representatives of each village participated in the study, the Catholic Church Health Services of Wewak Diocese, the Division of Health of East Sepik Province were also given.

\section{Funding}

This study was funded by JSPS KAKENHI Grant Number JP25305023 and by a research grant from TWMU Career Development Center for Medical Professionals, Tokyo Women's Medical University, Japan.

\section{Publisher's Note}

Springer Nature remains neutral with regard to jurisdictional claims in published maps and institutional affiliations.

Received: 19 July 2018 Accepted: 14 February 2019

Published online: 20 February 2019

References

1. WHO. Control and elimination of Plasmodium vivax: a technical brief. Geneva: World Health Organization; 2015. 
2. WHO. World malaria report. Geneva: World Health Organization; 2017.

3. Baird JK. Asia-Pacific malaria is singular, pervasive, diverse and invisible. Int J Parasitol. 2017:47:371-7.

4. Thriemer K, Ley B, Bobogare A, Dysoley L, Alam MS, Pasaribu AP, et al. Challenges for achieving safe and effective radical cure of Plasmodium vivax: a round table discussion of the APMEN Vivax Working Group. Malar J. 2017;16:141.

5. Takeuchi R, Lawpoolsri S, Imwong M, Kobayashi J, Kaewkungwal J, Pukrittayakamee S, et al. Directly-observed therapy (DOT) for the radical 14-day primaquine treatment of Plasmodium vivax malaria on the Thai-Myanmar border. Malar J. 2010;9:308.

6. Souares A, Lalou R, Sene I, Sow D, Le Hesran JY. Factors related to compliance to anti-malarial drug combination: example of amodiaquine/ sulphadoxine-pyrimethamine among children in rural Senegal. Malar J. 2009;8:118.

7. Almeida ED, Vieira JL. Factors associated with non-adherence to the treatment of vivax malaria in a rural community from the Brazilian Amazon Basin. Rev Soc Bras Med Trop. 2016:49:248-51.

8. Khantikul N, Butraporn P, Kim HS, Leemingsawat S, Tempongko MASB, Suwonkerd W. Adherence to antimalarial drug therapy among vivax malaria patients in Northern Thailand. J Health Popul Nutr. 2009;27:4-13.

9. Haque U, Scott LM, Hashizume M, Fisher E, Haque R, Yamamoto T, et al. Modelling malaria treatment practices in Bangladesh using spatial statistics. Malar J. 2012;11:63.

10. Chun CB, Kim SY, Lee YJ, Lee SY. Republic of Korea health system review. In: Health systems in transition. European Observatory on Health Systems and Policies. 2009;11:7.

11. The Ministry of Health Republic of Indonesia. National malaria control program strategic plan 2015-2019. Jakarta; 2015.

12. USAID. President's malaria initiative Thailand, Lao PDR, and regional malaria operational plan FY 2018: Washington, D.C., 2018.

13. Kingdom of Cambodia Ministry of Health. Cambodia malaria elimination action framework 2016-2020. Phnom Penh, 2016.

14. Phommanivong V, Thongkham K, Deyer G, Rene JP, Barennes H. An assessment of early diagnosis and treatment of malaria by village health volunteers in the Lao PDR. Malar J. 2010;9:347.

15. Tsukahara T, Ogura S, Sugahara T, Sekihara M, Furusawa T, Kondo N, et al. The choice of healthcare providers for febrile children after introducing non-professional health workers in a malaria endemic area in Papua New Guinea. Front Public Health. 2015:3:275.

16. Matsumoto-Takahashi EL, Kano S. Evaluating active roles of community health workers in accelerating universal access to health services for malaria in Palawan, the Philippines. Trop Med Health. 2016;44:10.

17. Morrow M, Nguyen QA, Caruana S, Biggs BA, Doan NH, Nong TT. Pathways to malaria persistence in remote central Vietnam: a mixed-method study of health care and the community. BMC Public Health. 2009;9:85.

18. Das LK, Jambulingam P, Sadanandane C. Impact of community-based presumptive chloroquine treatment of fever cases on malaria morbidity and mortality in a tribal area in Orissa State, India. Malar J. 2008;7:75.

19. Laverack $G$, Westberg $V$. Independent evaluation of a village health worker program. In: Vanuatu Village Health Worker Program. 2013. https ://dfat.gov.au/about-us/publications/Pages/independent-evaluation-ofthe-village-health-worker-program-vanuatu.aspx. Accessed 20 Dec 2018.

20. Mueller I, Widmer S, Michel D, Maraga S, McNamara DT, Kiniboro B, et al. High sensitivity detection of Plasmodium species reveals positive correlations between infections of different species, shifts in age distribution and reduced local variation in Papua New Guinea. Malar J. 2009;8:41.

21. Lin E, Kiniboro B, Gray L, Dobbie S, Robinson L, Laumaea A, et al. Differential patterns of infection and disease with P. falciparum and $P$. vivax in young Papua New Guinean children. PLoS ONE. 2010;5:e9047.

22. Grietens KP, Soto V, Erhart A, Ribera JM, Toomer E, Tenorio A, et al. Adherence to 7 day primaquine treatment for the radical cure of $P$. vivax in the Peruvian Amazon. Am J Trop Med Hyg. 2010;82:1017-23.

23. Karyana M, Devine A, Kenangalem E, Burdarm L, Poespoprodjo JR, Vemuri $\mathrm{R}$, et al. Treatment-seeking behaviour and associated costs for malaria in Papua, Indonesia. Malar J. 2016;15:536.

24. Pulford J, Kurumop SF, Ura Y, Siba PM, Mueller I, Hetzel MW. Malaria case management in Papua New Guinea following the introduction of a revised treatment protocol. Malar J. 2013;12:433.

25. Papua New Guinea National Department of Health. Human resources in child health. In: PNG Child Health and Policy Plan 2009-2020. https
://www.rch.org.au/uploadedFiles/Main/Content/cich/PNG_Child_Healt h_Policy_and_Plan_2009-2020.pdf. Accessed 20 Dec 2018.

26. O'Keefe D, Davis J, Yakuna G, Van Gemert C, Morgan C. Formal and informal maternal health care: comparing the service provision of health facilities and village health volunteers in East Sepik Province. PNG Med J. 2011;54:147-53.

27. Ashwell HE, Barclay L. Outcome evaluation of community health promotion intervention within a donor funded project climate in Papua New Guinea. Rural Remote Health. 2009:9:1219.

28. Inoue Y, Takahashi D, Kondo N, Yoshii A, Sekihara M, Hombhanje FW, et al. Village health volunteers' individual social capital and caretakers' health service utilization for febrile children in Malaria-endemic villages in Papua New Guinea. Trans R Soc Trop Med Hyg. 2017;111:490-6.

29. Genton B, Baea K, Lorry K, Ginny M, Wines B, Alpers MP. Parasitological and clinical efficacy of standard treatment regimens against Plasmodium falciparum, P. vivax and P. malariae in Papua New Guinea. P N G Med J. 2005;48:141-50.

30. Marfurt J, Mueller I, Sie A, Maku P, Goroti M, Reeder JC, et al. Low efficacy of amodiaquine or chloroquine plus sulfadoxine-pyrimethamine against Plasmodium falciparum and P. vivax malaria in Papua New Guinea. Am J Trop Med Hyg. 2007;77:947-54.

31. Karunajeewa HA, Mueller I, Senn M, Lin E, Law I, Gomorrai PS, et al. A trial of combination antimalarial therapies in children from Papua New Guinea. N Engl J Med. 2008;359:2545-57.

32. Papua New Guinea National Department of Health. National malaria treatment policy. Port Moresby: PNG NDoH; 2009.

33. Papua New Guinea National Department of Health. Standard treatment for common illness of adults in Papua New Guinea: a manual for nurses, community health workers, health extension officers and doctors. 5th ed. Port Moresby: PNG NDoH; 2003.

34. Divine Word University. Malaria diagnosis and the new treatment protocol, a training manual for health workers in Papua New Guinea. Port Moresby: Birdwing Publishing; 2010.

35. WHO. Guidelines for the treatment of malaria. 3rd ed. Geneva: World Health Organization; 2015.

36. Howe LD, Galobardes B, Matijasevich A, Gordon D, Johnston D, Onwujekwe $\mathrm{O}$, et al. Measuring socio-economic position for epidemiological studies in low- and middle-income countries: a methods of measurement in epidemiology paper. Int J Epidemiol. 2012;41:871-86.

37. Vyas S, Kumaranayake L. Constructing socio-economic status indices: how to use principal components analysis. Health Policy Plan. 2006;21:459-68.

38. Pereira EA, Ishikawa EA, Fontes CJ. Adherence to Plasmodium vivax malaria treatment in the Brazilian Amazon Region. Malar J. 2011;10:355.

39. Nyamongo IK. Health care switching behaviour of malaria patients in a Kenyan rural community. Soc Sci Med. 2002;54:377-86.

40. Müller O, Traoré C, Becher H, Kouyaté B. Malaria morbidity, treatmentseeking behaviour, and mortality in a cohort of young children in rural Burkina Faso. Trop Med Int Health. 2003;8:290-6.

41. Thandar MM, Kyaw MP, Jimba M, Yasuoka J. Caregivers' treatment-seeking behaviour for children under age five in malaria-endemic areas of rural Myanmar: a cross-sectional study. Malar J. 2015;14:1.

42. Getahun A, Deribe K, Deribew A. Determinants of delay in malaria treatment-seeking behaviour for under-five children in south-west Ethiopia: a case control study. Malar J. 2010;9:320.

43. Sowah LA, Turenne FV, Buchwald UK, Delva G, Mesidor RN, Dessaigne CG, et al. Influence of transportation cost on long-term retention in clinic for HIV patients in rural Haiti. J Acquir Immune Defic Syndr. 2014;67:e123-30.

44. Tuller DM, Bangsberg DR, Senkungu J, Ware NC, Emenyonu N, Weiser SD. Transportation costs impede sustained adherence and access to HAART in a clinic population in southwestern Uganda: a qualitative study. AIDS Behav. 2010;14:778-84.

45. Hung N, Hombhanje F, Eto H, Hwaihwanje I, Mita T, Tsukahara T, et al. Glucose-6-phosphate dehydrogenase (G6PD) variants in East Sepik Province of Papua New Guinea: G6PD Jammu, G6PD Vanua Lava, and a novel variant (G6PD Dagua). Trop Med Health. 2008;36:163-9.

46. Yenchitsomanus P, Summers KM, Chockkalingam C, Board PG. Characterization of G6PD deficiency and thalassaemia in Papua New Guinea. P N G Med J. 1986;29:53-8. 
47. Recht J, Ashley EA, White NJ. Use of primaquine and glucose-6-phosphate dehydrogenase deficiency testing: divergent policies and practices in malaria endemic countries. PLoS Negl Trop Dis. 2018;12:e0006230.

48. Nissen TN, Rose MV, Kimaro G, Bygbjerg IC, Mfinanga SG, Ravn P. Challenges of loss to follow-up in tuberculosis research. PLoS ONE. 2012;7:e40183.

49. Zachariah R, Harries AD, Manzi M, Gomani P, Teck R, Phillips M, et al. Acceptance of anti-retroviral therapy among patients infected with HIV and tuberculosis in rural Malawi is low and associated with cost of transport. PLOS ONE. 2006;1:e121.
50. Whorton CM, Yount E Jr. The Chesson strain of Plasmodium vivax malaria; clinical aspects. J Infect Dis. 1947;80:237-49.

51. Ministry of Health, Government of Vanuatu. Malaria diagnosis and treatment guideline, 2015. Port Vila; 2015.

52. Ingram RJ, Crenna-Darusallam C, Soebianto S, Noviyanti R, Baird JK. The clinical and public health problem of relapse despite primaquine therapy case review of repeated relapses of Plasmodium vivax acquired in Papua New Guinea. Malar J. 2014;13:488.

53. Tanahashi T. Health service coverage and its evaluatio. Bull World Health Organ. 1978;56:295-303.
Ready to submit your research? Choose BMC and benefit from:

- fast, convenient online submission

- thorough peer review by experienced researchers in your field

- rapid publication on acceptance

- support for research data, including large and complex data types

- gold Open Access which fosters wider collaboration and increased citations

- maximum visibility for your research: over 100M website views per year

At BMC, research is always in progress.

Learn more biomedcentral.com/submissions 